\title{
Avaliação comparativa da perda de extensão entre acessos cirúrgicos para revisão de artroplastia total do joelho*
}

\author{
Comparative assessment of extension loss among different \\ surgical approaches to the revision of total knee arthroplasty
}

\author{
Alfredo Maroues Villardi ${ }^{1}$, JoÃo Paulo Bezerra Leite², \\ FABIO MONTAGNER LEOMIL2${ }^{2}$, MARCELO MANDARINO ${ }^{3}$
}

\section{RESUMO}

Objetivo: Avaliar a possibilidade de perda de extensão do joelho, após artroplastias de revisão, realizadas com acessos do tipo quadriceps snip e osteotomia do tubérculo anterior da tíbia (TAT), comparativamente, entre ambos e com um grupo controle, em que foi utilizado o acesso parapatelar medial convencional. Método: Este estudo foi aprovado pelo Comitê de Ética em Pesquisa do INTO. A perda de extensão do joelho foi avaliada em pacientes submetidos a artroplastias de revisão, unilaterais, realizadas pelo Grupo de Joelho do Instituto Nacional de Traumatologia e Ortopedia - INTO, em que foram utilizados um dos seguintes acessos: convencional - parapatelar medial, snip do quadríceps ou

* Trabalho realizado no Instituto Nacional de Traumatologia e Ortopedia - INTO - Rio de Janeiro (RJ), Brasil.

1. Mestre, Pós-graduando do Programa de Pós-Graduação de Ciências Médicas da Universidade Estadual do Rio de janeiro - UERJ - Rio de Janeiro (RJ), Brasil; Membro do Grupo de Joelho do Instituto Nacional de Traumatologia e Ortopedia - INTO - Rio de Janeiro (RJ), Brasil.

2. Residentes do 3 o ano do Instituto Nacional de Traumatologia e Ortopedia - INTO - Rio de Janeiro (RJ), Brasil.

3. Médico Ortopedista filiado a clínica particular.

Endereço para correspondência: Alfredo Villardi, Av. Maracanã, 3.200/C01 - 20530-231 - Rio de Janeiro (RJ), Brasil. Tels.: (21)

2278-9177/9112-8511. E-mail: avillardi@ig.com.br

Recebido em 27/11/07. Aprovado para publicação em 24/4/08. Copyright RBO2008 osteotomia do tubérculo anterior da tíbia. A amostra foi composta por 26 indivíduos, cuja idade variou entre 52 e 80 anos, distribuídos em três grupos: grupo I: acesso parapatelar medial convencional ( $\mathrm{n}=10)$; grupo II: quadriceps snip $(\mathrm{n}=8)$; e grupo III: osteotomia do TAT $(n=8)$. $O$ tempo de seguimento médio foi de 18,57 meses. A perda da extensão do joelho foi determinada pela diferença entre o grau de extensão ativa pré e pós-operatória. Na avaliação estatística, foi utilizado o teste de análise de variância ANOVA, de fator único, com o nível de significância $\alpha=0,05$ (5\%). Resultados: A perda média de extensão da série foi igual a $8,57^{\circ}$, variando entre $2^{\circ} \mathrm{e} 30^{\circ}$. $\mathrm{O}$ p-valor obtido foi igual a 0,112, o que permite assumir a hipótese de igualdade entre os grupos. Conclusão: No presente estudo, não foi observada diferença significativa entre os grupos compostos pelos três tipos de acesso utilizados para as revisões de artroplastias, no que tange ao parâmetro perda de extensão.

Descritores - Cirurgia de Second-look; Artroplastia de joelho/métodos; Avaliação de resultado de intervenções terapêuticas; Estudos comparativos

\section{ABSTRACT}

Objective: To assess the possibility of extension loss of the knee after revision arthroplasties performed with approaches like quadriceps snip and TAT osteotomy, comparing the two methods and using a 
control group in who the conventional medial parapatellar approach was used. Method: This study was approved by the INTO Research Ethics Committee. The loss of extension of the knee was evaluated in patients submitted to unilateral revision arthroplasties of the knee conducted by the Knee Group of the INTO - National Institute of Traumatology and Orthopedics, the following approaches having been used: conventional - medial parapatellar, quadriceps snip, or anterior tibial tubercle osteotomy. The series included 26 individuals, ages ranging from 52 to 80, distributed into three groups: Group I: conventional medial parapatellar approach $(n=10)$; Group II: quadriceps snip $(n=8)$; and Group III: TAT osteotomy $(n=8)$. Mean follow-up was 18.57 months. Extension loss of the knee was determined by the difference between the range of active extension before and after surgery. In the statistical assessment, the ANOVA variance analysis test was used, with a single factor, with significance level $\alpha=0,05$ (5\%). Results: Mean extension loss in the series was equal to $8.57^{\circ}$, varying between $2^{\circ}$ and $30^{\circ}$. The p-value obtained was equal to 0.112, which leads to presume the hypothesis that groups are equal to one another. Conclusion: No significant difference was observed among the groups made up by the three types of approach used for arthroplasty revisions in terms of the loss of extension parameter.

\section{Keywords - Second-look surgery; Arthroplasty, replacement, knee/methods; Evaluation of results of therapeutic interventions; Comparative studies}

\section{INTRODUÇÃO}

A exposição cirúrgica adequada é de fundamental importância para realização da artroplastia de revisão. $\mathrm{O}$ acesso deve ser amplo o suficiente para permitir de forma apropriada a realização dos tempos cirúrgicos, como a remoção dos implantes, balanço ligamentar, manejo de perdas ósseas e re-implante dos componentes de revisão, além de minimizar o dano às partes moles e o risco de lesão do aparelho extensor.
Nos joelhos com arco de movimento inferior a $60^{\circ}$, a eversão patelar é dificultada devido à fibrose e aderências do coxim gorduroso peripatelar e retináculos ${ }^{(1-}$ 2). Se mesmo após a liberação não for obtida flexão maior que $90^{\circ}$ ou inversão patelar sem submeter a estresse excessivo a inserção do ligamento patelar, devese considerar a extensão do acesso proximalmente (através do tendão quadricipital e/ou retináculo lateral) ou distalmente (com a osteotomia do tubérculo anterior da tíbia - OTAT). Essa decisão deve ser baseada em diversos fatores: alongamento do mecanismo extensor, estoque ósseo tibial, necessidade de reposicionamento da patela, ou remoção da tíbia, hastes e cimento intramedular ${ }^{(2)}$.

Coonse et al descreveram um acesso que consiste em incisão em "V" invertido do tendão quadricipital avançando lateral e medialmente até o planalto tibial ${ }^{(3)}$.

Insall descreveu o patellar turndown, modificação do anterior, introduzindo um ramo lateral e distal à incisão parapatelar medial convencional, cruzando o tendão quadricipital sobre o retináculo lateral ${ }^{(4)}$. Essa modificação permite, como o original, exposição ampla, mas com vantagens teóricas, de preservar a artéria genicular súpero-lateral, além de possibilitar o alongamento do quadríceps (sutura em "Y") e de não violar $\mathrm{o}$ vasto medial. Entretanto, as maiores desvantagens desses acessos são a possibilidade de perda da extensão por necrose do ângulo proximal do retalho e/ou por alongamento do quadríceps, além de necessidade de proteção da sutura, com imobilização em extensão no pós-operatório ${ }^{(2,5)}$.

Barrack descreveu a liberação mínima do reto femoral - quadriceps snip - QS, como alternativa aos procedimentos de rebatimento distal do tendão quadricipital ${ }^{(5)}$. Consiste numa incisão oblíqua em $45^{\circ}$, estendendo-se superior e lateralmente, desde a extremidade proximal da incisão parapatelar medial capsular ao aspecto lateral do tendão quadricipital (figuras 1A e 1B).

A OTAT foi descrita inicialmente por Dolin e modificada por Whiteside ${ }^{(6-7)}$. Consiste em osteotomia transversa completa da crista tibial estendendo-se distalmente, por aproximadamente $10 \mathrm{~cm}$, desde o ápice do 


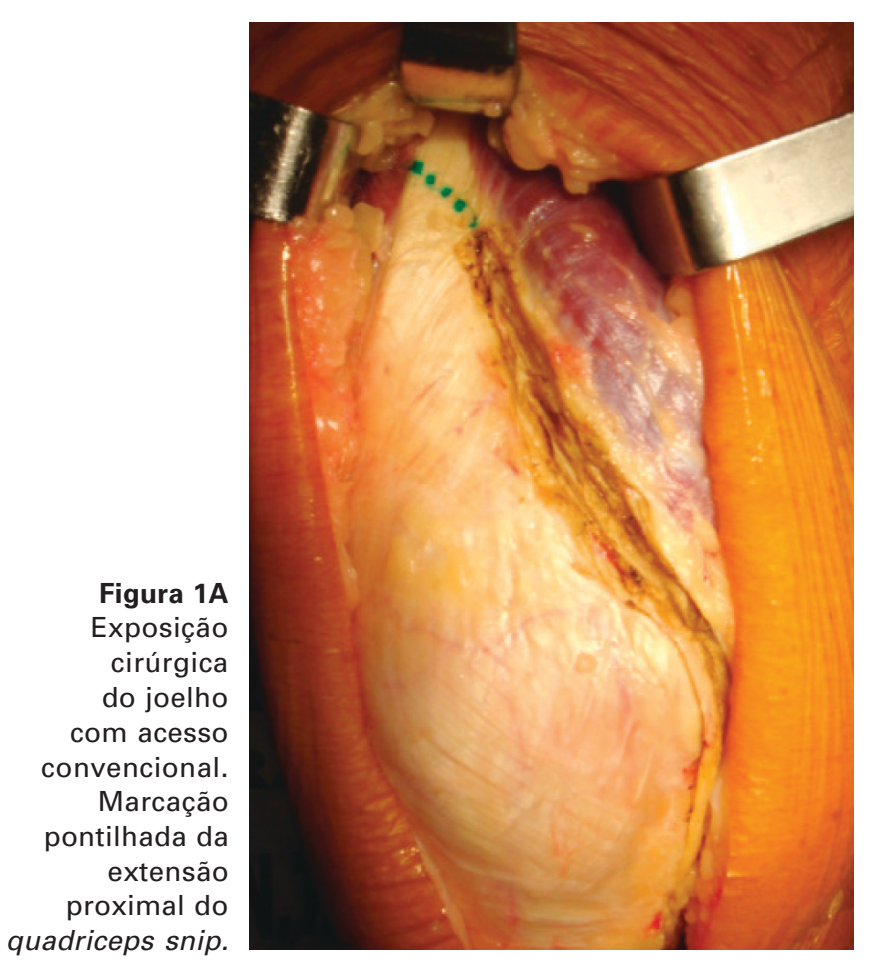

tubérculo tibial. Os tecidos moles inseridos na região são deixados intactos, sendo rebatidos lateralmente juntamente com o mecanismo extensor ${ }^{(7)}$. Assim, ocorre violação mínima do suprimento vascular regional (figura 2). O fragmento ósseo pode ser reinserido por meio de parafusos ou amarrias ${ }^{(8)}$ (figuras 3 e 4 ). A osteotomia curta deve ser evitada, devido à associação com altas taxas de complicações ${ }^{(1,9)}$.

A OTAT possibilita excelente exposição de joelhos rígidos, tendo suas principais indicações nos casos de patela baixa, em que se pode fixar proximalmente o fragmento ósseo ou, de acordo com a necessidade, de uma janela para o canal medular da tíbia, útil para remoção de cimento ósseo ou de implantes tibiais de haste longa ${ }^{(1)}$. A utilização dessa técnica é dependente do estoque ósseo adequado da porção proximal da tíbia.

Diversos estudos relatam perda de extensão do joelho, com diferentes acessos utilizados em artroplastias de revisão ${ }^{(2,7,9-11)}$.

O objetivo deste estudo é avaliar a possibilidade de perda de extensão do joelho, após artroplastias de revisão, realizadas com acessos do tipo QS e OTAT, com-

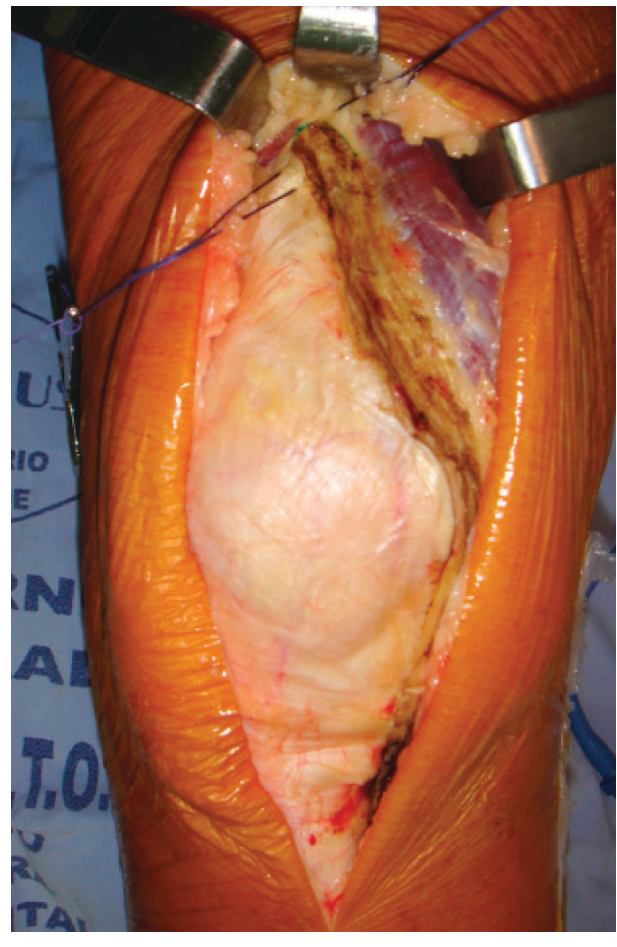

Figura 1B

Exposição cirúrgica do joelho. Incisão sobre o tendão do quadríceps sobre a marcação pontilhada. Quadriceps snip.

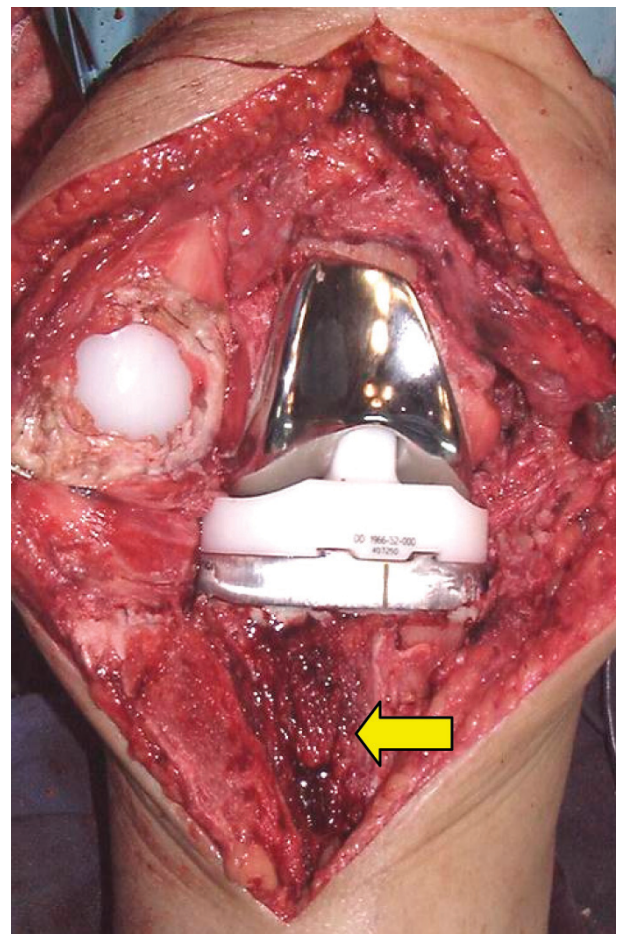

Figura 2

Artroplastia de revisão com osteotomia do TAT. Seta apontando para o leito da osteotomia tibial.

parativamente, entre ambos e com um grupo controle, em que foi utilizado o acesso parapatelar medial convencional. 


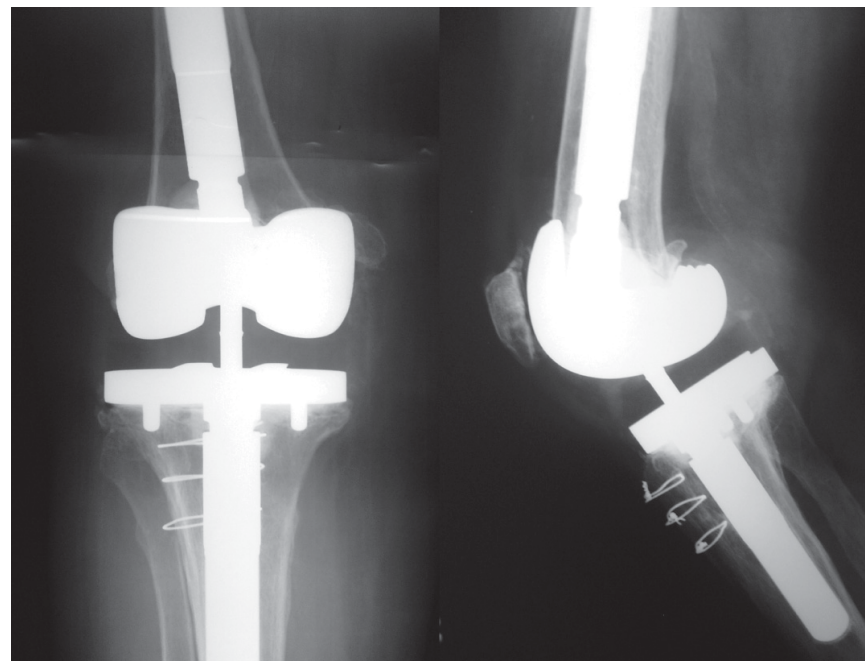

Figura 3 - Radiografias em AP e lateral de artroplastia de revisão com osteotomia do TAT, fixada com cerclagem tripla

\section{MÉTODO}

O presente estudo foi submetido à avaliação e aprovado pelo Comitê de Ética em Pesquisa do Instituto Nacional de Traumatologia e Ortopedia.

No período entre janeiro de 2002 e dezembro de 2004, foram realizadas pelo grupo de Joelho do Instituto Nacional de Traumatologia e Ortopedia 54 artroplastias de revisão do joelho, todas unilaterais.

Foram estudados 26 pacientes submetidos à revisão asséptica de artroplastia do joelho, em que foram utilizados um dos seguintes acessos: convencional - parapatelar medial, QS ou OTAT, que apresentavam pelo menos seis meses de pós-operatório no momento da avaliação, com programa de reabilitação concluído, sem história de perda de extensão do joelho pré-operatória ou de rotura do aparelho extensor.

A exclusão dos outros 28 casos deveu-se a um ou à combinação de mais de um dos seguintes fatores: tratar-se revisão séptica, tanto de um quanto dois estágios, distúrbios neurovasculares, presença de contratura em flexão, reabilitação incompleta, uso de espaçador para tratamento de infecção, perda de seguimento, não comparecimento à avaliação, casos de re-revisão ou por não ter sido utilizado nas revisões algum dos acessos de interesse da pesquisa.

A amostra foi composta por sete indivíduos do sexo masculino $(26,92 \%)$ e 19 do feminino $(73,08 \%)$, com

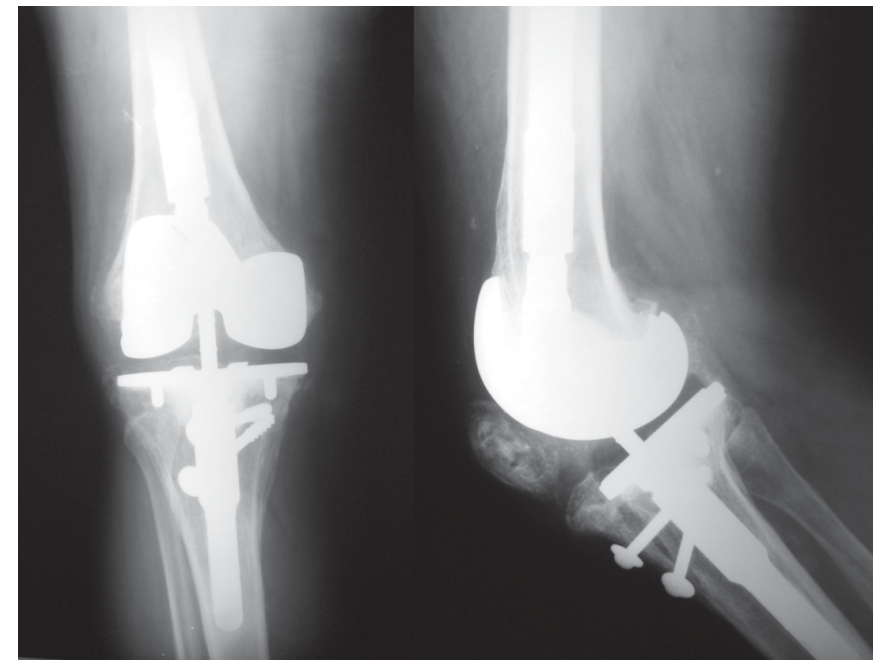

Figura 4 - Radiografias em AP e lateral de artroplastia de revisão com osteotomia do TAT, fixada com parafusos e arruelas

idade variando entre 52 e 80 anos (média = 68,92 anos), distribuídos em três grupos: grupo I: acesso parapatelar medial convencional ( $\mathrm{n}=10$ ); grupo II: quadriceps snip $(\mathrm{n}=8)$; e grupo III: osteotomia do TAT ( $\mathrm{n}=$ 8) (tabelas 1, 2 e 3 ).

O tempo de seguimento médio foi de 18,85 meses, sendo o maior igual a 40 meses e o menor, igual a sete meses.

A opção de utilizarem-se os acessos QS ou OTAT não esteve condicionada a maior ou menor gravidade do caso operado. Não houve, portanto, hierarquização de procedimentos, ou seja, os casos menos complexos serem abordados com um acesso e os mais complexos com outro.

A extensão ativa foi mensurada por meio de goniometria analógica, com escala de $1^{\circ}$. Com o paciente colocado na posição sentada, solicitava-se que realizasse extensão ativa do joelho contra a gravidade, sendo, então, mensurado o grau de extensão ativa (figura 5).

$\mathrm{O}$ arco de movimento passivo foi analisado com o paciente em decúbito dorsal, realizando a extensão e a flexão máxima possível até encontrar resistência articular.

As mensurações da extensão ativa foram realizadas por um único avaliador e a da amplitude articular passiva, por dois avaliadores, para padronização da coleta de dados. 
TABELA 1

Sujeitos do grupo I - acesso convencional

\begin{tabular}{rcccc}
\hline Caso no & Idade & Sexo & $\begin{array}{c}\text { Tempo de } \\
\text { seguimento } \\
\text { (em meses) }\end{array}$ & $\begin{array}{r}\text { Perda da } \\
\text { extensão ativa } \\
\text { (em graus) }\end{array}$ \\
\hline 1 & 79 & $\mathrm{~F}$ & 9 & $4^{\circ}$ \\
2 & 65 & $\mathrm{M}$ & 33 & $10^{\circ}$ \\
3 & 65 & $\mathrm{~F}$ & 9 & $2^{\circ}$ \\
4 & 74 & $\mathrm{~F}$ & 13 & $8^{\circ}$ \\
5 & 76 & $\mathrm{~F}$ & 10 & $4^{\circ}$ \\
6 & 72 & $\mathrm{~F}$ & 8 & $2^{\circ}$ \\
7 & 67 & $\mathrm{~F}$ & 36 & $3^{\circ}$ \\
8 & 76 & $\mathrm{~F}$ & 10 & $5^{\circ}$ \\
9 & 59 & $\mathrm{M}$ & 22 & $5^{\circ}$ \\
10 & 52 & $\mathrm{~F}$ & 7 & $10^{\circ}$ \\
\hline
\end{tabular}

Fonte: INTO 2003-2007

TABELA 2

Sujeitos do grupo II - quadríceps snip

\begin{tabular}{ccccc}
\hline Caso no & Idade & Sexo & $\begin{array}{c}\text { Tempo de } \\
\text { seguimento } \\
\text { (em meses) }\end{array}$ & $\begin{array}{c}\text { Perda da } \\
\text { extensão ativa } \\
\text { (em graus) }\end{array}$ \\
\hline 1 & 68 & $\mathrm{~F}$ & 24 & $15^{\circ}$ \\
2 & 74 & $\mathrm{M}$ & 18 & $10^{\circ}$ \\
3 & 68 & $\mathrm{M}$ & 8 & $30^{\circ}$ \\
4 & 72 & $\mathrm{~F}$ & 19 & $5^{\circ}$ \\
5 & 61 & $\mathrm{~F}$ & 32 & $14^{\circ}$ \\
6 & 60 & $\mathrm{~F}$ & 8 & $30^{\circ}$ \\
7 & 76 & $\mathrm{~F}$ & 24 & $0^{\circ}$ \\
8 & 73 & $\mathrm{~F}$ & 22 & $2^{\circ}$ \\
\hline
\end{tabular}

Fonte: INTO 2003-2007

TABELA 3

Sujeitos do grupo III - osteotomia do tubérculo anterior da tíbia

\begin{tabular}{ccccc}
\hline Caso no & Idade & Sexo & $\begin{array}{c}\text { Tempo de } \\
\text { seguimento } \\
\text { (em meses) }\end{array}$ & $\begin{array}{c}\text { Perda da } \\
\text { extensão ativa } \\
\text { (em graus) }\end{array}$ \\
\hline 1 & 59 & M & 11 & $0^{\circ}$ \\
2 & 80 & $\mathrm{~F}$ & 36 & $20^{\circ}$ \\
3 & 61 & $\mathrm{~F}$ & 30 & $0^{\circ}$ \\
4 & 79 & $\mathrm{~F}$ & 40 & $10^{\circ}$ \\
5 & 67 & $\mathrm{M}$ & 19 & $5^{\circ}$ \\
6 & 74 & $\mathrm{~F}$ & 13 & $5^{\circ}$ \\
7 & 70 & $\mathrm{M}$ & 13 & $14^{\circ}$ \\
8 & 65 & $\mathrm{~F}$ & 9 & $10^{\circ}$ \\
\hline
\end{tabular}

Fonte: INTO 2003-2007

Rev Bras Ortop. 2008;43(4):126-32

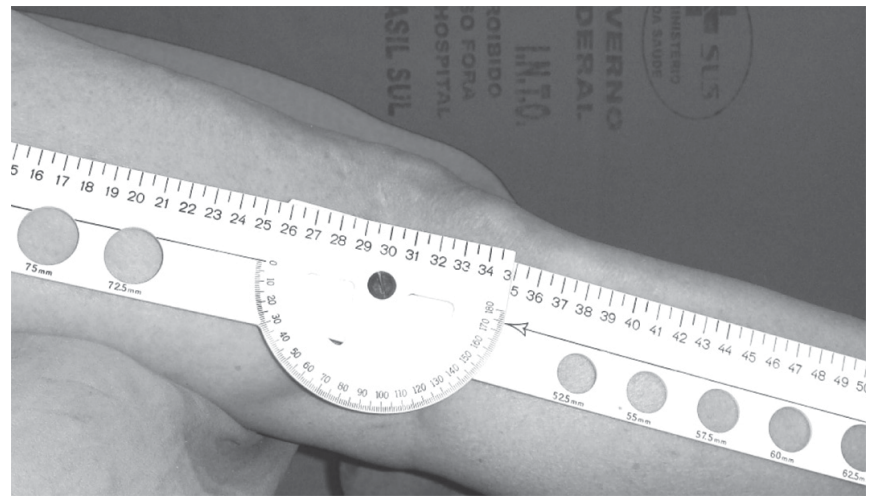

Figura 5 - Determinação goniométrica da extensão ativa do joeIho

A perda da extensão do joelho foi determinada pela diferença entre o grau de extensão pré e pós-operatória, obtida na avaliação de cada paciente. Os valores pré-operatórios foram coletados nos prontuários.

Para análise estatística, foi utilizado o teste de análise de variância ANOVA, de fator único, com o nível de significância $\alpha=0,05$ (5\%). Com base em valores representativos de situação de existência de diferença entre os grupos (hipótese alternativa verdadeira), obteve-se estimativa de tamanho amostral de 13 indivíduos para cada grupo para se obter "poder de teste" de $80 \%$ com nível de significância (alfa) de 5\%. Com o número de indivíduos empregados no presente trabalho, para o mesmo nível de significância de 5\%, o "poder de teste" atingido foi de $61 \%$. Entretanto, a redução do teste (sobre a probabilidade de se cometer o "erro tipo 1"), com o emprego de alfa de $10 \%$, os resultados continuaram indicando a aceitação da hipótese nula, de não haver diferença entre os três grupos (valor-p $=11,2 \%$ ), mas com "poder de teste" de $74 \%$, valor que já traria maior confiabilidade a essa inferência.

A média de idade da série foi de 68,92 anos. A observada entre os indivíduos estudados nos três grupos foi de 68,50 anos no grupo I, de 69,00 anos no grupo II e de 69,37 no grupo III.

O tempo de seguimento nos grupos I, II e III foi igual a 15,70 meses $(\mathrm{DP} \pm 10,7), 19,37$ meses (DP \pm $8,1)$ e de 21,37 meses $(\mathrm{DP} \pm 12,1)$, respectivamente (gráfico 1). 


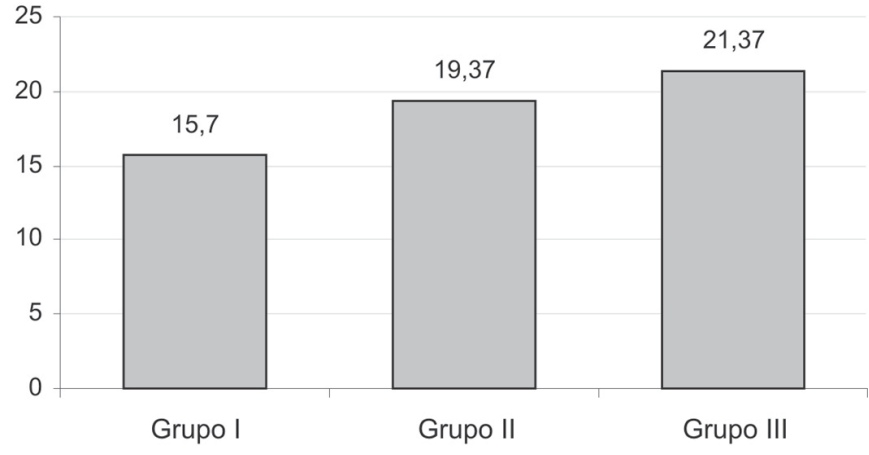

Gráfico 1 - Tempo de seguimento pós-operatório de cada grupo Fonte: INTO 2003-2007

\section{RESULTADOS}

A perda da extensão, considerando-se os três grupos, variou entre $2^{\circ}$ e $30^{\circ}$, com média igual a $8,57^{\circ}$ (DP $\pm 8,1$ ).

A amplitude média de movimento passivo nos três grupos foi igual a $93,88^{\circ}$, variando entre $30^{\circ}$ e $150^{\circ}$.

No grupo I, a perda de extensão média foi igual a $5,3^{\circ}$, variando entre $2^{\circ}$ e $10^{\circ}(\mathrm{DP} \pm 3,0)$. No grupo II, a perda de extensão média foi igual a $13,25^{\circ}$ e variou entre $2^{\circ}$ e $30^{\circ}$ (DP $\left.\pm 11,6\right)$. No grupo III a perda máxima foi de $20^{\circ}$, a mínima de $5^{\circ}$ e a média igual a $8^{\circ}$ (DP $\pm 6,9$ ) (gráfico 2).

Nenhum caso de pseudartrose foi observado nos pacientes submetidos à OTAT.

$\mathrm{O}$ p-valor obtido foi igual a 0,112 , o que permite assumir a hipótese de que não há diferença estatisticamente significativa entre os grupos.

A maior variância foi observada no grupo II $(135,07)$ e o de menor variância foi o grupo I, $(9,12)$, ficando o grupo II com a posição intermediária $(47,71)$.

\section{DISCUSSÃO}

Em uma artroplastia de revisão, não raras vezes, com o acesso convencional, parapatelar medial, é difícil obter exposição cirúrgica adequada, sem que se coloque em risco a integridade do aparelho extensor, em especial quando há flexão limitada do joelho.

Dessa forma, é necessária a utilização de alternativas de acesso que possam permitir a lateralização ou inversão patelar e a devida proteção do aparelho extensor.

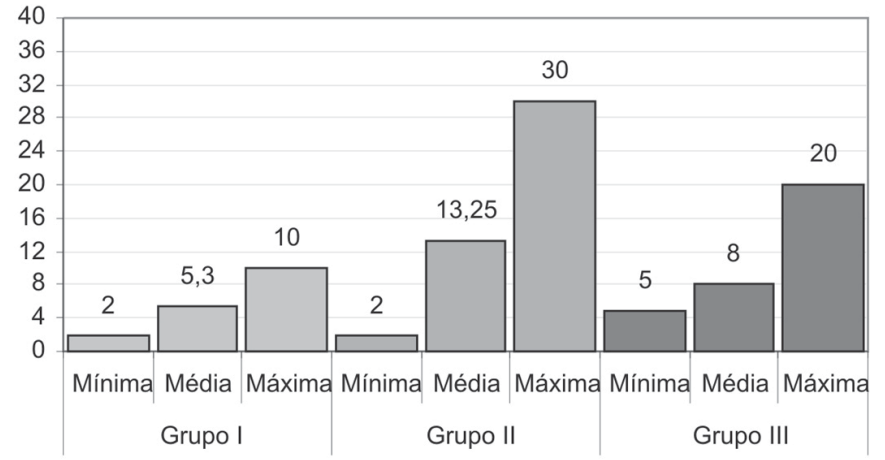

Gráfico 2 - Índice de perda de extensão em cada grupo Fonte: INTO 2003-2007

Dentre as diversas alternativas de prolongamento do acesso convencional, pretendemos avaliar, comparativamente, neste estudo, uma proximal - QS e outra distal - OTAT, no que tange, especificamente, à perda de extensão do quadríceps. Garvin et al avaliaram uma série de casos de revisão em que foi utilizado o QS e observaram, na avaliação isocinética, perda de força de extensão nos joelhos revisados, em comparação com os contralaterais normais. Entretanto, nenhuma diferença foi observada, ao comparar joelhos revisados com o do lado oposto, também operados. Afirmaram, portanto, que o QS consiste em técnica segura, simples e que não necessita de instrumental especial para sua realização, além de não haver necessidade de modificação na reabilitação pós-operatória( ${ }^{(2)}$. Patel et al não observaram perda de extensão em 81 joelhos submetidos à revisão com o $\mathrm{QS}^{(12)}$. Estudos comparativos concluíram que não houve diferença nos diversos parâmetros estudados entre os acessos convencionais e os QS, utilizados em artroplastias de revisão(11,13). No nosso estudo, embora o número amostral tenha sido menor do que ambos, também não foi observada diferença estatística, de perda de extensão, ao comparar esses dois tipos de exposição.

Whiteside afirmou que as forças de tensão sobre o tendão quadricipital durante a extensão do joelho são maiores que sobre o tendão patelar. Por isso, recomendou que a intervenção sobre o mecanismo extensor distalmente seria mais benéfica ${ }^{(7)}$.

Maruyama afirma que, nos casos estudados de revisões com OTAT, todos consolidaram e que não foram 
observadas complicações relacionadas com a técnica empregada ${ }^{(14)}$. Mendes et al relataram 7\% de complicações, diretamente relacionadas com a OTAT ${ }^{(15)}$. Outros estudos não detectaram perda de extensão do joelho em artroplastias com o emprego da OTAT $^{(16-17)}$. Apenas dois casos de perda de extensão foram relatados no trabalho de Whiteside, casos que se mantiveram inalterados em relação a sua condição pré-operatória, num total de 136 cirurgias com o recurso da $\mathrm{OTAT}^{(7)}$. Em outro estudo, foi identificada incidência de $8 \%$ de perda de extensão em revisões com OTAT, valor comparável ao do grupo em que foi utilizado o quadriceps turndown ${ }^{(12)}$.

No nosso estudo, também encontramos casos de perda de extensão em revisões com OTAT. Em números absolutos, a perda média de extensão foi menor no

\section{REFERÊNCIAS}

1. Rand JA, Ries MD, Landis GH, Rosenberg AG, Haas S. Intraoperative assessment in revision total knee arthroplasty. J Bone Joint Surg Am. 2003;85-A Suppl 1:S26-37.

2. Garvin KL, Scuderi G, Insall JN. Evolution of the quadriceps snip. Clin Orthop Relat Res. 1995;(321):131-7.

3. Coonse K, Adams JD. A new operative approach to the knee joint. Surg Gynecol Obstet. 1943;77:344-7.

4. Insall JN. Surgical approaches. In: Insall JE, Windsor RE, Scott WN, Kelly MA, Aglietti P, editors. Surgery of the knee. 2a ed. New York; Edinburgh: Churchill Livingstone; 1993. p.135-48.

5. Barrack RL. Instructional Course Lectures, The American Academy of Orthopaedic Surgeons: Specialized exposure for revision total knee arthroplasty: Quadriceps snip and patellar turndown. J Bone Joint Surg Am. 1999;81(1):138-41.

6. Dolin MG. Osteotomy of the tibial tubercle in total knee replacement. A technical note. J Bone Joint Surg Am. 1983; 65(5):704-6. grupo I (acesso convencional), seguido pelo grupo III (OTAT) e, finalmente, pelo grupo II (QS), o que não se traduziu em diferença estatisticamente significativa entre os três grupos estudados.

Reconhecemos o limitado número amostral do nosso estudo e, portanto, consideramos plenamente justificável a realização de outras pesquisas que possam contribuir com novas evidências do impacto funcional produzido em artroplastias de revisões com acessos alternativos.

\section{CONCLUSÃO}

No presente estudo, não foi observada diferença entre os grupos compostos pelos três tipos de acesso utilizados para as revisões de artroplastias, no que tange ao desfecho perda de extensão.

7. Whiteside LA. Exposure in difficult total knee arthroplasty using tibial tubercle osteotomy. Clin Orthop Relat Res. 1995; (321):32-5.

8. Wolff AM, Hungerford DS, Krackow KA, Jacobs MA. Osteotomy of the tibial tubercle during total knee replacement. A report of twenty-six cases. J Bone Joint Surg Am. 1989; 71(6):848-52.

9. Trousdale RT, Hanssen AD, Rand JA, Cahalan TD. V-Y quadricepsplasty in total knee arthroplasty. Clin Orthop Relat Res. 1993;(286):48-55.

10. Mullen JO. Range of motion following total knee arthroplasty in ankylosed joints. Clin Orthop Relat Res. 1983;(179):200-3.

11. Barrack RL, Smith P, Munn B, Engh G, Rorabeck C. The Ranawat Award. Comparison of surgical approaches in total knee arthroplasty. Clin Orthop Relat Res. 1998;(356):16-21.

12. Patel JV, Masonis JL, McCalden R, MacDonald S, Bourne RB, Rorabeck CH. Functional outcome of extensile exposures used in revision knee arthroplasty. J Bone Joint Surg Br. 2003;85-B Suppl 2:166. 